\title{
Upper bound estimate of character sums over Lehmer's numbers
}

Di Han and Wenpeng Zhang*

${ }^{\text {"Correspondence: }}$

wpzhang@nwu.edu.cn

Department of Mathematics,

Northwest University, Xi'an, Shaanxi, P.R. China

\section{Abstract}

Let $p$ be an odd prime. For each integer $a$ with $1 \leq a \leq p-1$, it is clear that there exists one and only one $\bar{a}$ with $0 \leq \bar{a} \leq p-1$ such that $a \cdot \bar{a} \equiv 1 \bmod p$. Let $\mathcal{A}$ denote the set of all integers $1 \leq a \leq p-1$, in which $a$ and $\bar{a}$ are of opposite parity. The main purpose of this paper is using the analytic method and the properties of Kloosterman sums to study the estimate problem of the mean value $\sum_{a \in \mathcal{A}} \chi(a)$, and give a sharp upper bound estimate for it, where $\chi$ denotes any non-principal even character mod $p$.

MSC: $11 L 40$

Keywords: Lehmer's numbers; character sums; Kloosterman sums; upper bound estimate

\section{Introduction}

Let $q \geq 3$ be an odd number. For each integer $a$ with $1 \leq a \leq q-1$ and $(a, q)=1$, it is clear that there exists one and only one $b$ with $0 \leq b \leq q-1$ such that $a b \equiv 1 \bmod q$. Let $\mathcal{A}(q)=\mathcal{A}$ denote the set of cases, in which $a$ and $b$ are of opposite parity. For $q=p$, an odd prime, professor Lehmer [1] asked to study $|\mathcal{A}|$ or at least to say something nontrivial about it, where $p$ is a prime, and $|\mathcal{A}|$ denote the number of all elements in $\mathcal{A}$. We call such a number a Lehmer's number. It is known that $|\mathcal{A}| \equiv 2$ or $0 \bmod 4$ when $p \equiv \pm 1 \bmod 4$. For general odd number $q \geq 3$, Zhang ([2] and [3]) studied the asymptotic properties of $|\mathcal{A}|$, and obtained a sharp asymptotic formula for it. That is, he proved the asymptotic formula

$$
|\mathcal{A}|=\frac{1}{2} \phi(q)+O\left(q^{\frac{1}{2}} \cdot d^{2}(q) \cdot \ln ^{2} q\right)
$$

where $\phi(q)$ is the Euler function, and $d(q)$ is the Dirichlet divisor function.

Let $M(a, p)$ denote the number of all integers $1 \leq b, c \leq p-1$ such that $b c \equiv a \bmod p$ and $(2, b+c)=1$, and $E(a, p)=M(a, p)-\frac{p-1}{2}$. Then Zhang [4] also studied the mean value properties of $E(a, p)$, and proved that

$$
\sum_{a=1}^{p-1} E^{2}(a, p)=\frac{3}{4} p^{2}+O\left(p \cdot \exp \left(\frac{3 \ln p}{\ln \ln p}\right)\right),
$$

where $\exp (y)=e^{y}$. Some related works can also be found in [5, 6] and [7].

\section{Springer}

(c) 2013 Han and Zhang; licensee Springer. This is an Open Access article distributed under the terms of the Creative Commons Attribution License (http://creativecommons.org/licenses/by/2.0), which permits unrestricted use, distribution, and reproduction in any medium, provided the original work is properly cited. 
In this paper, we consider the estimate problem of the character sums

$$
\sum_{a \in \mathcal{A}} \chi(a)=\sum_{\substack{a=1 \\ 2 \uparrow a+\bar{a}}}^{p-1} \chi(a)
$$

and give a sharper upper bound estimate for it, where $\chi$ denotes any non-principal even character $\bmod p$.

About character sums (1.1), it is clear that its value is zero, if $\chi$ is an odd character mod $p$. If $\chi$ is a non-principal even character mod $p$, then how large is the upper bound estimate of (1.1)? About this problem, it seems that none had studied it yet, at least we have not seen any related results. The problem is interesting, because it can help us to understand the deep properties of the character sums over some special sets, for example, Lehmer's numbers.

The main purpose of this paper is using the analytic method and the properties of Kloosterman sums to study this problem, and prove the following result.

Theorem Let $p>3$ be an odd prime. Then for any non-principal character $\chi \bmod p$, we have the estimate

$$
\sum_{a \in \mathcal{A}} \chi(a) \ll p^{\frac{1}{2}} \cdot \ln ^{2} p
$$

For general odd number $q \geq 3$, whether there exists a similar upper bound estimate for (1.1) is an interesting problem, we will further study it.

\section{Several lemmas}

In this section, we will give several lemmas, which are necessary in the proof of our theorem. Hereinafter, we will use many properties of character sums, all of which can be found in [8], so they will not be repeated here. First we have the following.

Lemma 1 Let $q>2$ be an odd number. Suppose that $\chi$ is an odd character mod $q$, then we have the identity

$$
(1-2 \chi(2)) \sum_{a=1}^{q} a \chi(a)=\chi(2) q \sum_{a=1}^{\frac{q-1}{2}} \chi(a)
$$

Proof See reference [9].

Lemma 2 Let $q>2$ be an odd number. Then for any even character $\chi_{1} \bmod q$, we have the identity

$$
\sum_{a \in \mathcal{A}} \chi(a)=-\frac{2}{\phi(q)} \sum_{\substack{\chi \bmod q \\ \chi(-1)=-1}}\left(1-2 \chi \chi_{1}(2)\right)(1-2 \chi(2))\left(\frac{1}{q} \sum_{a=1}^{q} a \chi \chi_{1}(a)\right)\left(\frac{1}{q} \sum_{a=1}^{q} a \chi(a)\right)
$$

where $\sum_{\chi(-1)=-1} \underset{x \bmod q}{ }$ denotes the summation over all odd characters $\chi \bmod q$. 
Proof From the orthogonality relation for character sums $\bmod q$ and the definition of $\mathcal{A}$ we have

$$
\begin{aligned}
\sum_{a \in \mathcal{A}} \chi(a) & =\frac{1}{2} \sum_{a=1}^{q-1}\left(1-(-1)^{a+\bar{a}}\right) \chi_{1}(a)=\frac{1}{2} \sum_{a=1}^{q-1} \chi_{1}(a)-\frac{1}{2} \sum_{a=1}^{q-1}(-1)^{a+\bar{a}} \chi_{1}(a) \\
& =-\frac{1}{2 \phi(q)} \sum_{\chi \bmod q}\left(\sum_{a=1}^{q-1}(-1)^{a} \chi_{1} \chi(a)\right)\left(\sum_{b=1}^{q-1}(-1)^{b} \chi(b)\right),
\end{aligned}
$$

where $\sum_{a=1}^{\prime q-1}$ denotes the summation over all $1 \leq a \leq q$ such that $(a, q)=1$.

If $\chi(-1)=1$, then

$$
\sum_{b=1}^{q-1}(-1)^{b} \chi(b)=0 .
$$

If $\chi(-1)=-1$, then

$$
\sum_{b=1}^{q-1}(-1)^{b} \chi(b)=2 \chi(2) \sum_{b=1}^{\frac{q-1}{2}} \chi(b) .
$$

Note that if $\chi(-1)=-1$ and $\chi_{1}(-1)=1$, then $\chi \chi_{1}(-1)=-1$. So combining (2.1), (2.2), (2.3) and Lemma 1, we have

$$
\begin{aligned}
\sum_{a \in \mathcal{A}} \chi(a) & =-\frac{1}{2 \phi(q)} \sum_{\substack{\chi \bmod q \\
\chi(-1)=-1}}\left(2 \chi \chi_{1}(2) \sum_{a=1}^{(q-1) / 2} \chi_{1} \chi(a)\right)\left(2 \chi(2) \sum_{b=1}^{(q-1) / 2} \chi(b)\right) \\
& =-\frac{2}{\phi(q)} \sum_{\substack{\chi \bmod q \\
\chi(-1)=-1}}\left(1-2 \chi \chi_{1}(2)\right)(1-2 \chi(2))\left(\frac{1}{q} \sum_{a=1}^{q} a \chi \chi_{1}(a)\right)\left(\frac{1}{q} \sum_{a=1}^{q} a \chi(a)\right) .
\end{aligned}
$$

This proves Lemma 2.

Lemma 3 Let $p$ be an odd prime, let $\chi$ be any character $\bmod p$. Then for any integers $m$ and $n$, we have the estimate

$$
\sum_{a=1}^{p-1} \chi(a) e\left(\frac{m a+n \bar{a}}{p}\right) \ll(m, n, p)^{\frac{1}{2}} \cdot p^{\frac{1}{2}}
$$

where $e(y)=e^{2 \pi i y},(m, n, p)$ denotes the greatest common divisor of $m, n$ and $p$.

Proof See [10] and [11].

Lemma 4 Let $p$ be an odd prime, let $\chi$ be any even character mod $p$. Then for any integer $c$ with $(c, p)=1$, we have the estimate

$$
\left|\sum_{\substack{\chi \bmod p \\ \chi(-1)=-1}} \chi(c) \tau(\chi) \tau\left(\chi \chi_{1}\right) L(1, \overline{\chi \chi}) L(1, \bar{\chi})\right| \ll p^{\frac{3}{2}} \ln ^{2} p .
$$


Proof For any non-principal character $\chi \bmod p$, applying Abel's identity (see Theorem 4.2 of [8]), we have

$$
L\left(1, \overline{\chi \chi} \chi_{1}\right) L(1, \bar{\chi})=\sum_{n=1}^{p^{3}} \frac{\bar{\chi}(n) \sum_{d \mid n} \bar{\chi}_{1}(d)}{n}+\int_{p^{3}}^{\infty} \frac{A(y, \bar{\chi})}{y^{2}} d y,
$$

where $A(y, \bar{\chi})=\sum_{p^{3}<n \leq y} \bar{\chi}(n) \sum_{d \mid n} \bar{\chi}_{1}(d)$.

From [12], we know that for any real number $y>p^{3}$, we have the estimate

$$
\sum_{\substack{\chi \bmod p \\ \chi(-1)=-1}}|A(y, \bar{\chi})|^{2} \ll y \phi^{2}(p)
$$

From (2.4), (2.5), Lemma 3, the orthogonality relation for character sums mod $p$, the definition of Gauss sums, and noting that $|\tau(\chi)|=\sqrt{p}$, we have

$$
\begin{aligned}
& \sum_{\substack{\chi \bmod p \\
\chi(-1)=-1}} \chi(c) \tau(\chi) \tau\left(\chi \chi_{1}\right) L(1, \overline{\chi \chi}) L(1, \bar{\chi}) \\
& =\sum_{a=1}^{p-1} \sum_{b=1}^{p-1} \chi_{1}(a) e\left(\frac{a+b}{p}\right) \sum_{n=1}^{p^{3}} \frac{\sum_{d \mid n} \bar{\chi}_{1}(d)}{n} \sum_{\substack{\chi \bmod p \\
\chi(-1)=-1}} \chi(a b c \bar{n}) \\
& +O\left(\sum_{\chi \bmod p}|\tau(\chi)| \cdot\left|\tau\left(\chi \chi_{1}\right)\right| \int_{p^{3}}^{\infty} \frac{|A(y, \bar{\chi})|}{y^{2}} d y\right) \\
& =\frac{p-1}{2} \sum_{n=1}^{p^{3}} \frac{\sum_{d \mid n} \bar{\chi}_{1}(d)}{n} \sum_{\substack{a=1 \\
a b c \equiv n \bmod p}}^{p-1} \sum_{\substack{b=1 \\
\text { mod }}}^{p-1} \chi_{1}(a) e\left(\frac{a+b}{p}\right) \\
& -\frac{p-1}{2} \sum_{n=1}^{p^{3}} \frac{\sum_{d \mid n} \bar{\chi}_{1}(d)}{n} \sum_{\substack{a=1 \\
a b c \equiv-n \bmod p}}^{p-1} \sum_{\substack{p-1 \\
a c}}^{p} \chi_{1}(a)\left(\frac{a+b}{p}\right) \\
& +O\left(p \cdot \sum_{\substack{\chi \bmod p \\
\chi(-1)=-1}} \int_{p^{3}}^{\infty} \frac{|A(y, \bar{\chi})|}{y^{2}} d y\right) \\
& =\frac{p-1}{2} \sum_{n=1}^{p^{3}} \frac{\sum_{d \mid n} \bar{\chi}_{1}(d)}{n} \sum_{a=1}^{p-1} \chi_{1}(a) e\left(\frac{a+n \bar{c} \cdot \bar{a}}{p}\right) \\
& -\frac{p-1}{2} \sum_{n=1}^{p^{3}} \frac{\sum_{d \mid n} \bar{\chi}_{1}(d)}{n} \sum_{a=1}^{p-1} \chi_{1}(a) e\left(\frac{a-n \bar{c} \cdot \bar{a}}{p}\right)+O(p) \\
& =O\left(p^{\frac{3}{2}} \sum_{n=1}^{p^{3}} \frac{d(n)}{n}\right)=O\left(p^{\frac{3}{2}} \cdot \ln ^{2} p\right) .
\end{aligned}
$$

This proves Lemma 4. 


\section{Proof of the theorems}

In this section, we will complete the proof of our theorem. First, if $\chi(-1)=-1$, then from Theorems 12.11 and 12.20 of [8], we have

$$
\frac{1}{p} \sum_{b=1}^{p} b \chi(b)=\frac{i}{\pi} \tau(\chi) L(1, \bar{\chi})
$$

From (3.1), Lemma 2 and Lemma 4, we have

$$
\begin{aligned}
& \sum_{a \in \mathcal{A}} \chi(a)=-\frac{2}{\phi(p)} \sum_{\substack{\chi \bmod p \\
\chi(-1)=-1}}\left(1-2 \chi \chi_{1}(2)\right)(1-2 \chi(2)) \\
& \times\left(\frac{1}{p} \sum_{a=1}^{p} a \chi \chi_{1}(a)\right)\left(\frac{1}{p} \sum_{a=1}^{p} a \chi(a)\right) \\
& =\frac{2}{\pi^{2}(p-1)} \sum_{\substack{\chi \bmod p \\
\chi(-1)=-1}}\left(1-2 \chi \chi_{1}(2)\right)(1-2 \chi(2)) \tau(\chi) \tau\left(\chi \chi_{1}\right) L\left(1, \bar{\chi} \times \chi_{1}\right) L(1, \bar{\chi}) \\
& =\frac{2}{\pi^{2}(p-1)} \sum_{\substack{\chi \bmod p \\
\chi(-1)=-1}} \tau(\chi) \tau\left(\chi \chi_{1}\right) L\left(1, \overline{\chi \chi_{1}}\right) L(1, \bar{\chi}) \\
& -\frac{4}{\pi^{2}(p-1)} \sum_{\substack{\chi \bmod p \\
\chi(-1)=-1}} \chi(2) \tau(\chi) \tau\left(\chi \chi_{1}\right) L\left(1, \overline{\chi \chi_{1}}\right) L(1, \bar{\chi}) \\
& -\frac{4}{\pi^{2}(p-1)} \chi_{1}(2) \sum_{\substack{\chi \bmod p \\
\chi(-1)=-1}} \chi(2) \tau(\chi) \tau\left(\chi \chi_{1}\right) L\left(1, \overline{\chi \chi_{1}}\right) L(1, \bar{\chi}) \\
& +\frac{8}{\pi^{2}(p-1)} \chi_{1}(2) \sum_{\substack{\chi \bmod p \\
\chi(-1)=-1}} \chi(4) \tau(\chi) \tau\left(\chi \chi_{1}\right) L\left(1, \overline{\chi \chi_{1}}\right) L(1, \bar{\chi}) \\
& =O\left(\frac{1}{p} \cdot p^{\frac{3}{2}} \cdot \ln ^{2} p\right)=O\left(p^{\frac{1}{2}} \cdot \ln ^{2} p\right) \text {. }
\end{aligned}
$$

This completes the proof of our theorem.

\section{Competing interests}

The authors declare that they have no competing interests.

\section{Authors' contributions}

DH studied the estimate problem of the mean value $\sum_{a \in \mathcal{A}} \chi(a)$, and gave a sharp upper bound estimate for it. WZ participated in the research and summary of the study.

\section{Acknowledgements}

The authors would like to thank the referees for their very helpful and detailed comments, which have significantly improved the presentation of this paper. This work is supported by the N.S.F. $(11071194,11001218)$ of P.R. China and G.I.C.F. (YZZ12062) of NWU

Received: 8 July 2013 Accepted: 7 August 2013 Published: 20 August 2013

\section{References}

1. Guy, RK: Unsolved Problems in Number Theory, 2nd edn. Springer, New York (1994)

2. Zhang, W: On a problem of D. H. Lehmer and its generalization. Compos. Math. 86, 307-316 (1993)

3. Zhang, W: A problem of D. H. Lehmer and its generalization (II). Compos. Math. 91, 47-56 (1994) 
4. Zhang, W: A problem of D. H. Lehmer and its mean square value formula. Jpn. J. Math. 29, 109-116 (2003)

5. Ping, X, Yi, Y: On character sums over flat numbers. J. Number Theory 130, 1234-1240 (2010)

6. Zhang, W: A mean value related to D. H. Lehmer's problem and the Ramanujan's sum. Glasg. Math. J. 54, 155-162 (2012)

7. Zhang, W: On the difference between an integer and its inverse modulo $n$ (II). Sci. China Ser. A 46, $229-238$ (2003)

8. Apostol, TM: Introduction to Analytic Number Theory. Springer, New York (1976)

9. Funakura, T: On Kronecker's limit formula for Dirichlet series with periodic coefficients. Acta Arith. 55, 59-73 (1990)

10. Chowla, S: On Kloosterman's sums. Norske Vid. Selsk. Forh. 40, 70-72 (1967)

11. Malyshev, AV: A generalization of Kloosterman sums and their estimates. Vestn. Leningr. Univ. 15, $59-75$ (1960) (in Russian)

12. Zhang, W: On the mean values of Dedekind sums. J. Théor. Nr. Bordx. 8, 429-442 (1996)

doi:10.1186/1029-242X-2013-392

Cite this article as: Han and Zhang: Upper bound estimate of character sums over Lehmer's numbers. Journal of Inequalities and Applications 2013 2013:392.

\section{Submit your manuscript to a SpringerOpen ${ }^{\odot}$ journal and benefit from:}

- Convenient online submission

- Rigorous peer review

- Immediate publication on acceptance

- Open access: articles freely available online

- High visibility within the field

- Retaining the copyright to your article 